\title{
Supersymmetry in Quantum Mechanics of Colored Particles
}

\author{
Sh. Mamedov, ${ }^{\text {a,b }}$ 网 Jian-zu Zhanga,cin and V. Zhukovskiid \\ a) Department of Physics, University of Kaiserslautern, 67653 \\ Kaiserslautern, Germany \\ b) High Energy Physics Laboratory, Baku State University, 23 Z. Khalilov, \\ 370148 Baku, Azerbaijan \\ c) Institute for Theoretical Physics, Box 316, East China University of \\ Science and Technology, Shanghai 200237, P.R. China \\ d) Faculty of Physics, Department of Theoretical Physics, Moscow State \\ University, 119899 Moscow, Russia
}

\begin{abstract}
The role of supercharge operators is studied in the case of a Dirac particle moving in a constant chromomagnetic field. The Hamiltonian is factorised and the ground state wave function in the case of unbroken supersymmetry is determined.
\end{abstract}

The study of supersymmetric quantum mechanics began with ref. [1] and has been applied to various problems, including in particular the case of charged abelian particles [2, 3, 4, 5, 6, 6]. In refs. [2] and [3] the supersymmetry of the equation of motion of the electron in a magnetic field was investigated. The related problem of supersymmetry of the Dirac equation for a colored particle in an external chromonagnetic field has been considered in ref. [8]. In the following the investigation of the latter is continued with the aim to explore the significance of the supercharge operators in this case of a chromomagnetic field, to factorise the Hamiltonian and to determine the ground state wave function.

It is known [9, 10] that a constant color background can be obtained from two types of vector potentials $A_{\mu}$, i.e. linearly growing abelian potentials and

\footnotetext{
* Email:shahin@physik.uni-kl.de

†Email:jzzhang@physik.uni-kl.de

†Email:th180@phys.msu.su
} 
non-commuting vector potentials. Here we consider the simplest case of the latter type which implies the non-commuting potentials

$$
\begin{aligned}
& A_{\mu}=A_{\mu}^{a} T^{a}=A_{\mu}^{a} \tau^{a} / 2, \quad a=1,2,3, \quad \mu=0,1,2,3, \\
& A_{\mu}^{1}=\left(0, \sqrt{\lambda_{1}}, 0,0\right), A_{\mu}^{2}=\left(0,0, \sqrt{\lambda_{2}}, 0\right), A_{\mu}^{3}=0 .
\end{aligned}
$$

with $\sqrt{\lambda_{1}}, \sqrt{\lambda_{2}}$ constant. Latin indices are $S U(2)$ color indices and Greek indices are Lorentz ones. The Pauli matrices $\tau_{i}$ are the generators of the $S U(2)$ color group. The potentials (1) determine a constant homogeneous chromomagnetic field along the third axes of both ordinary and color spaces, i.e.

$$
F_{12}^{3}=g \epsilon^{a b 3} A_{1}^{a} A_{2}^{b}=g \sqrt{\lambda_{1} \lambda_{2}}=H_{z}^{3}
$$

where $g$ is the constant of color interaction. With analogous non-commuting potentials a constant field could be given for any chromomagnetic or chromoelectric component.

The Dirac equation for a colored particle in the chromomagnetic field (1) is

$$
\left[\gamma_{\mu}\left(p_{\mu}+g A_{\mu}^{a} \frac{\tau^{a}}{2}\right)-m\right] \psi=0
$$

In terms of Majorana spinors $\phi$ and $\chi \psi=\left(\begin{array}{c}\phi \\ \chi\end{array}\right)$ and with $P_{\mu}=p_{\mu}+g A_{\mu}^{a} \tau^{a} / 2$ we rewrite eq.(3) as

$$
\begin{aligned}
\sigma_{i} P_{i} \chi & =\left(i \frac{\partial}{\partial t}-m\right) \phi \\
\sigma_{i} P_{i} \phi & =\left(i \frac{\partial}{\partial t}+m\right) \chi
\end{aligned}
$$

Here the Pauli matrices $\sigma_{i}$ describe the particle's spin. The spinors $\phi$ and $\chi$ can easily be seen to satisfy the equations

$$
\begin{aligned}
& (\sigma \cdot \mathbf{P})^{2} \phi=-\left(\frac{\partial^{2}}{\partial t^{2}}+m^{2}\right) \phi \\
& (\sigma \cdot \mathbf{P})^{2} \chi=-\left(\frac{\partial^{2}}{\partial t^{2}}+m^{2}\right) \chi
\end{aligned}
$$

Setting

$$
Q=\sigma \cdot \mathbf{P}
$$

and

$$
H=-\left(\frac{\partial^{2}}{\partial t^{2}}+m^{2}\right)
$$

eqs. (5) assume the form

$$
Q^{2} \psi=H \psi
$$

The "Hamiltonian" (7) (cf. ref. [2]) has eigenvalues $E^{2}-m^{2}$, i.e.

$$
H \psi=\left(E^{2}-m^{2}\right) \psi \text {. }
$$


Eqs. (8) are reminiscent of Witten's one-measure supersymmetric quantum mechanics [1] for $N$ supercharges. In this the supersymmetry algebra has the form

$$
\left\{Q_{i}, Q_{j}\right\}=2 \delta_{i j} H, \quad\left[H, Q_{i}\right]=0, \quad i=1,2,3, \cdots N
$$

where the curly bracket denotes an anticommutator. In view of its commutation with $H$ the quantity $\sigma \cdot \mathbf{P}$ is conserved on the classical level. It is known in supersymmetric theories that supercharge operators $Q_{i}$ obeying (10) lead to a degeneracy of the energy. The relation between the degeneracy $n$ and the number of supercharges $N$ is given by the formula

$$
n=2^{[N / 2]}
$$

where $[N / 2]$ means integer part of $N / 2$. The energy spectrum of a colored particle in the field (11) was found in [10 and is given by

$$
E_{1,2}^{2}=\mathbf{p}^{2}+m^{2}+\frac{g^{2}\left(\lambda_{1}+\lambda_{2}\right)}{4} \pm g \sqrt{\lambda_{1} p_{1}^{2}+\lambda_{2} p_{2}^{2}+\frac{\left(H_{z}^{3}\right)^{2}}{4}}
$$

This spectrum is doubly degenerate so that two energy levels correspond to four states having different quantum numbers of $(\sigma, \tau)$.

From the explicit expression of the operator $Q$ of eq.(6) it is seen that supersymmetry transformations transform a state with quantum numbers $(p, \sigma, \tau)$ into another state with quantum numbers $\left(p^{\prime \prime}, \sigma^{\prime \prime}, \tau^{\prime \prime}\right)$. There are states with the same energy among these. The states with the same energy are called superpartners. There are therefore superpartner states on the field of eq.(四). According to eq.(11) there is a second supercharge operator in the theory. Since $A_{3}^{a}=0$ the $\mathrm{z}$-dependence of the quark wave function is of the plane wave type. Considering the case $p_{z}=0$ at initial time $t=0$, it is reasonable to consider the supercharge $Q$ in the $(x, y)$ plane and set

$$
Q_{1}=\sigma_{1} P_{1}+\sigma_{2} P_{2}
$$

The second supercharge operator can be constructed with the help of the prescription suggested in ref.[2]:

$$
Q_{2}=i Q_{1} \sigma_{3}=\sigma_{2} P_{1}-\sigma_{1} P_{2}
$$

The supercharge $Q_{2}$ is hermitian and together with $Q_{1}$ obeys Witten's supercharge algebra eq. (10). With the help of $Q_{1}$ and $Q_{2}$ we can construct hermitian mutually conjugate supercharge operators $Q_{ \pm}$by setting

$$
Q_{ \pm}=\frac{1}{2}\left(Q_{1} \pm i Q_{2}\right)=Q_{1} \frac{1 \mp \sigma_{3}}{2}
$$

These operators obey the field theory supersymmetry algebra 11 and the Jacobi identity:

$$
\begin{aligned}
Q_{+}^{2} & =Q_{-}^{2}=0, \quad\left\{Q_{+}, Q_{-}\right\}=H, \quad\left[Q_{ \pm}, H\right]=0 \\
0 & \equiv\left\{\left[Q_{+}, H\right], Q_{-}\right\}+\left\{\left[Q_{-}, H\right], Q_{+}\right\}+\left[H,\left\{Q_{+}, Q_{-}\right\}\right]
\end{aligned}
$$


Setting

$$
P_{ \pm}=P_{1} \pm i P_{2}, \quad a_{ \pm}=\frac{1}{2}\left(\sigma_{1} \pm i \sigma_{2}\right),
$$

which satisfy $P_{ \pm}^{\dagger}=P_{\mp}, a_{ \pm}^{\dagger}=a_{\mp}$, the operators $Q_{ \pm}$can be expressed as

$$
Q_{+}=P_{-} a_{+}, \quad Q_{-}=P_{+} a_{-},
$$

and the supersymmetry algebra (15) is fulfilled by the relations

$$
\left[Q_{+}, P_{-}\right]=0, \quad\left[Q_{-}, P_{+}\right]=0, \quad\left\{Q_{+}, a_{-}\right\}=P_{-}, \quad\left\{Q_{-}, a_{+}\right\}=-P_{+},
$$

which is in full agreement with ref.[11. Only four of the six operators are independent.

It is easy to see from the definition of the operators $a_{ \pm}$that they obey the algebra of creation and annihilation operators for fermion degrees of freedom, i.e.

$$
\left\{a_{+}, a_{-}\right\}=1, \quad\left(a_{+}\right)^{2}=\left(a_{-}\right)^{2}=0 .
$$

This implies that the fermion degree of freedom in the quantum mechanics of the nonabelian charged particle in a chromomagnetic field is the particle's spin. The commutator of the operators $P_{ \pm}$gives

$$
\left[P_{+}, P_{-}\right]=g H_{z}^{3} \tau_{3}
$$

The spinors $\phi$ and $\chi$ of eqs. (4) and (5) have two components corresponding to different projections of spin which we write $\psi=\left(\begin{array}{c}\psi_{+} \\ \psi_{-}\end{array}\right)$. The components $\psi_{+}$and $\psi_{-}$are spinors in color space which we write $\psi_{+}=\left(\begin{array}{c}\psi_{+}^{(1)} \\ \psi_{+}^{(2)}\end{array}\right), \psi_{-}=$ $\left(\begin{array}{c}\psi_{-}^{(1)} \\ \psi_{-}^{(2)}\end{array}\right)$. Since the color operator $\tau_{3}$ has its own two eigenvalues, the commutator (19) splits into two commutation relations depending on the color state chosen, i.e.

$$
\begin{array}{ll}
\text { a) } \quad\left[P_{+}, P_{-}\right] & =g H_{z}^{3} \quad \text { for state } \psi_{ \pm}^{(1)} \text { with } \quad \hat{\tau}_{3} \psi_{ \pm}^{(1)}=\psi_{ \pm}^{(1)} \\
\text { b) } \quad\left[P_{+}, P_{-}\right] & =-g H_{z}^{3} \quad \text { for state } \psi_{ \pm}^{(2)} \text { with } \hat{\tau}_{3} \psi_{ \pm}^{(2)}=-\psi_{ \pm}^{(2)}
\end{array}
$$

We run into a situation which is analogous to one in ref. 12 where the quantum mechanical motion of a wave packet was shown to be made up of a mixture of states with $\tau_{3}$-eigenvalues $=+1$ and -1 . In a chromomagnetic field such a packet breaks up into modes with different values of $\tau_{3}$ moving in opposite directions. We introduce operators $b_{ \pm}$as creation and annihilation operators of bosonic states respectively by defining

$$
b_{ \pm}=\frac{P_{ \pm}}{\sqrt{g H_{z}^{3}}}
$$

which obey correspondingly the Heisenberg-Weyl algebra

$$
\text { a) }\left[b_{+}, b_{-}\right]=1 \text { for } \psi_{ \pm}^{(1)}, \quad \text { b) }\left[b_{-}, b_{+}\right]=1 \text { for } \psi_{ \pm}^{(2)} .
$$


¿From this we see, that the operators $b_{+}$and $b_{-}$interchange their roles for state $\psi^{(1)}$ and state $\psi^{(2)}$. The meaning of the operators $Q_{ \pm}$of eqs.(16) becomes clear *: If $n_{f}$ and $n_{b}$ denote the number of fermions and bosons respectively, the operator $Q_{+}$transforms a state $\psi^{(1)}$ with $\left(n_{f}, n_{b}\right)$ into the state $\psi^{(2)}$ with $\left(n_{f}+1, n_{b}-1\right)$ and the state $\psi^{(2)}$ with $\left(n_{f}+1, n_{b}-1\right)$ into the state $\psi^{(1)}$ with $\left(n_{f}, n_{b}\right)$, and the operator $Q_{-}$transforms the state $\psi^{(2)}$ with $\left(n_{f}+1, n_{b}-1\right)$ into the state $\psi^{(1)}$ with $\left(n_{f}, n_{b}\right)$ and the state $\psi^{(1)}$ with $\left(n_{f}, n_{b}\right)$ into the state $\psi^{(2)}$ with $\left(n_{f}+1, n_{b}-1\right)$. In any case the sum of fermion and boson numbers is conserved, $n_{f}+n_{b}=$ const.

We now rewrite the anticommutator (16) using (17):

$$
H=Q_{+} Q_{-}+Q_{-} Q_{+}=\frac{1}{2}\left\{P_{-}, P_{+}\right\}+\frac{1}{2}\left[P_{-}, P_{+}\right] \sigma_{3}=\left(\begin{array}{cc}
P_{-} P_{+} & 0 \\
0 & P_{+} P_{-}
\end{array}\right)
$$

Thus the Hamiltonian of (8) is split into two parts corresponding to different projections of spin. According to eq. (9) we have

$$
H \psi=\left(\begin{array}{cc}
P_{-} P_{+} & 0 \\
0 & P_{+} P_{-}
\end{array}\right) \psi=\left(E^{2}-m^{2}\right)\left(\begin{array}{c}
\psi_{+} \\
\psi_{-}
\end{array}\right)
$$

Thus we have two independent equations:

$$
\begin{aligned}
& P_{-} P_{+} \psi_{+}=\left(E^{2}-m^{2}\right) \psi_{+}, \\
& P_{+} P_{-} \psi_{-}=\left(E^{2}-m^{2}\right) \psi_{-}
\end{aligned}
$$

These equations could be used for finding the ground state wave functions of quarks in the field (1). It is well known that with unbroken supersymmetry the ground state has zero energy eigenvalue. As we see from (12) the energy spectrum $E_{1}^{2}$ has its minimal eigenvalue at $\mathbf{p}=0$,

$$
\left(E_{1}^{2}\right)_{\min }=\left(m^{2}+\frac{g^{2}\left(\lambda_{1}^{1 / 2}+\lambda_{2}^{1 / 2}\right)^{2}}{4}\right)
$$

and the spectrum $E_{2}^{2}$ has its minimal eigenvalue at $\mathbf{p}=\left(0, g \sqrt{\left(\lambda_{2}-\lambda_{1}\right)} / 2,0\right)$ for $\lambda_{2}>\lambda_{1}$ and at $\mathbf{p}=\left(g \sqrt{\left(\lambda_{1}-\lambda_{2}\right)} / 2,0,0\right)$ for $\lambda_{1}>\lambda_{2}$ with

$$
\left(E_{2}^{2}\right)_{\min }=m^{2} \text {. }
$$

Hence only for the spectrum $E_{2}^{2}$ supersymmetry is unbroken for massless quarks $(m=0)$. Then for the ground state with unbroken supersymmetry eqs.(24) assume the form

$$
P_{-} P_{+} \psi_{+}=0, \quad P_{+} P_{-} \psi_{-}=0 .
$$

These equations show that the Hamiltonian for the ground state is reduced to a product of two linear, i.e. factorised, operators. According to (27) the expectation values of the operators $P_{\mp} P_{ \pm}$vanish, i.e.

$$
<\psi_{+}\left|P_{-} P_{+}\right| \psi_{+}>=0, \quad<\psi_{-}\left|P_{+} P_{-}\right| \psi_{-}>=0
$$

${ }^{*}$ Here the operators $a_{ \pm}$do not have the same meaning as in field theory where the fermion operators imply changes of spin by $\pm 1 / 2$. 
Since $P_{ \pm}$are mutually hermitian conjugate operators these equations can be written as

$$
\left|P_{+}\right| \psi_{+}>\left.\right|^{2}=0,\left|P_{-}\right| \psi_{-}>\left.\right|^{2}=0 .
$$

Thus to find the ground state wave function we have to solve the two linear equations obtained from (29). Taking into account the explicit expressions of $P_{ \pm}$and the color components $\psi_{ \pm}^{(1),(2)}$ of the spinors $\psi_{ \pm}$, we obtain

$$
\begin{aligned}
& P_{+} \mid \psi_{+}>=\left(\begin{array}{cc}
p_{1}+i p_{2} & \frac{i g}{2}\left(A_{1}^{1}+A_{2}^{2}\right) \\
\frac{i g}{2}\left(A_{1}^{1}-A_{2}^{2}\right) & p_{1}+i p_{2}
\end{array}\right)\left(\begin{array}{c}
\psi_{+}^{(1)} \\
\psi_{+}^{(2)}
\end{array}\right)=0, \\
& P_{-} \mid \psi_{+}>=\left(\begin{array}{cc}
p_{1}-i p_{2} & \frac{i g}{2}\left(A_{1}^{1}-A_{2}^{2}\right) \\
\frac{i g}{2}\left(A_{1}^{1}+A_{2}^{2}\right) & p_{1}-i p_{2}
\end{array}\right)\left(\begin{array}{c}
\psi_{-}^{(1)} \\
\psi_{-}^{(2)}
\end{array}\right)=0 .
\end{aligned}
$$

These equations are easily solved in polar coordinates with $x=r \cos \theta, y=$

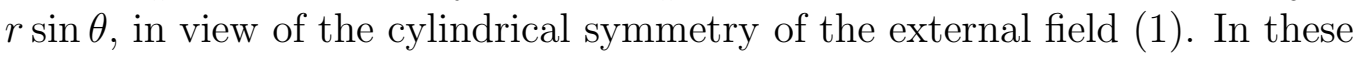
coordinates the operators $p_{1} \pm i p_{2}$ assume the form

$$
p_{1} \pm i p_{2}=e^{ \pm i \theta}\left(\frac{\partial}{\partial r} \mp \frac{i}{r} \frac{\partial}{\partial \theta}\right)
$$

We thus have two independent variables $r, \theta$ and one constraint resulting from conservation of $Q=\sigma \cdot \mathbf{P}$. We choose the reference frame so that $\theta$ is the angle between $\sigma$ and $\mathbf{P}$, and we assume that the external chromomagnetic field given by potentials (1) is given in this reference frame, since otherwise, on passing to any other moving frame, the external field (11) will also possess chromoelectric components. Conservation of $\sigma \cdot \mathbf{P}$ means $\cos \theta=$ const. Consequently $\theta=$ const. and $\partial \psi_{ \pm}^{(1),(2)} / \partial \theta=0$. With this eqs.(30) assume the well known form

$$
\left(\frac{\partial^{2}}{\partial r^{2}}+\frac{g^{2}}{4} e^{\mp 2 i \theta}\left(\lambda_{2}-\lambda_{1}\right)\right) \psi_{ \pm}^{(1)}=0, \quad \psi_{ \pm}^{(2)}=\frac{2 e^{ \pm i \theta}}{g\left(\sqrt{\lambda_{1}}+\sqrt{\lambda_{2}}\right)} \frac{\partial}{\partial r} \psi_{ \pm}^{(1)} .
$$

Setting

$$
\xi=\frac{g}{2} \sqrt{\lambda_{2}-\lambda_{1}} \sin \theta, \quad \eta=\frac{g}{2} \sqrt{\lambda_{2}-\lambda_{1}} \cos \theta,
$$

the general solution of eqs. (32) can be written

$$
\begin{aligned}
& \psi_{+}^{(1)}=C_{1} e^{\xi r} e^{i \eta r}+C_{2} e^{-\xi r} e^{-i \eta r}, \\
& \psi_{+}^{(2)}=i \sqrt{\frac{\sqrt{\lambda_{2}}-\sqrt{\lambda_{1}}}{\sqrt{\lambda_{1}}+\sqrt{\lambda_{2}}}}\left(C_{1} e^{\xi r} e^{i \eta r}-C_{2} e^{-\xi r} e^{-i \eta r}\right), \\
& \psi_{-}^{(1)}=i \sqrt{\frac{\sqrt{\lambda_{2}}-\sqrt{\lambda_{1}}}{\sqrt{\lambda_{1}}+\sqrt{\lambda_{2}}}}\left(C_{1}^{\prime} e^{-\xi r} e^{i \eta r}-C_{2}^{\prime} e^{\xi r} e^{-i \eta r}\right), \\
& \psi_{-}^{(2)}=C_{1}^{\prime} e^{-\xi r} e^{i \eta r}+C_{2}^{\prime} e^{\xi r} e^{-i \eta r} .
\end{aligned}
$$

Selection of the normalizable parts depends on whether $\lambda_{2}>$ or $\left\langle\lambda_{1}\right.$ and on the value of the angle $\theta$. For instance when $\lambda_{2}>\lambda_{1}$ and $0<\theta \leq \pi / 2$, we 
have

$$
\begin{aligned}
& \psi_{+}^{(1)}=C_{2} e^{-\xi r} e^{-i \eta r} \\
& \psi_{+}^{(2)}=-i C_{2} \sqrt{\frac{\sqrt{\lambda_{2}}-\sqrt{\lambda_{1}}}{\sqrt{\lambda_{1}}+\sqrt{\lambda_{2}}}} e^{-\xi r} e^{-i \eta r} \\
& \psi_{-}^{(1)}=i C_{1}^{\prime} \sqrt{\frac{\sqrt{\lambda_{2}}-\sqrt{\lambda_{1}}}{\sqrt{\lambda_{1}}+\sqrt{\lambda_{2}}}} e^{-\xi r} e^{i \eta r} \\
& \psi_{-}^{(2)}=C_{1}^{\prime} e^{-\xi r} e^{i \eta r} .
\end{aligned}
$$

Further analysis of the Dirac equation shows that there is no supersymmetry in the chromoelectric case. Also supersymmetry is broken in a chromomagnetic field with spherically symmetric components.

\section{Acknowledgments}

Sh. M. and J.-Z. Z. acknowledge support by DAAD and discussions with H. J. W. Müller-Kirsten. JZZ's work has also been supported by the National Natural Science Foundation of China under the grant number 10074014 and by the Shanghai Education Development Foundation. 


\section{References}

[1] E. Witten, Nucl. Phys. B188 (1981) 513.

[2] L. Gendenshtein and I. Krive, Uspekhi Fizicheskikh Nauk 146 (1985) 552 [Sov. Phys. Usp. 28 (1985)645].

[3] L. Gendenshtein, J. of Nucl. Phys. 41(1985) 261.

[4] Yu. R. Musin, Sov. J. Phys.5 (1990) 28.

[5] A.V. Smilga, Nucl. Phys. B249 (1985) 413.

[6] P. Solomonson and I.W. van Holten, Nucl. Phys. B196 (1982) 509.

[7] F. Cooper, A. Khare and U. Sukhatme, Phys. Rept. 251 (1995) 267, hep-th/ 9405029; A.Das and S.A. Pernice, Mod. Phys. Lett. A12 (1997) 581, hep-th/9612125.

[8] V. C. Zhukovskii, JETP 90 (1986) 1137.

[9] L.S. Brown and W. J. Weisberger, Nucl. Phys. B157 (1979) 285.

[10] M. Reuter and C. Wetterich, Phys. Lett. B334 (1994) 412, hep$\mathrm{ph} / 9405300$.

[11] I. M. Ternov, V. C. Zhukovskii and A. V. Borisov, Quantum Processes in Strong External Fields, M. Nauka (1981).

[12] V. Ogievetskii and L. Mezinchesku, Uspekhi Fizicheskikh Nauk 117 (1975) 673 [Sov. Phys. Usp. 18 (1975) 960].

[13] V.C. Zhukovskii and S. A. Mamedov, Soviet J. Phys. 1 (1990) 106. 